\title{
EDITORIAL
}

\section{Disruption of the lung structure maintenance programme: a comprehensive view of emphysema development}

\author{
J.A. Barberà*,\# and V.I. Peinado*,\#
}

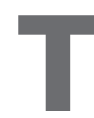
he lung is highly exposed to the external environment and must cope with a number of challenges from inhaled particles, toxic gases and invading microorganisms. Defence against these external injuries depends on various mechanisms (antioxidants, antiproteases and the immune response), and an efficient system for removing and replacing apoptotic cells. Failure to cope with external injury may lead to lung damage and destruction.

Emphysema is a characteristic feature in the lungs of patients with chronic obstructive pulmonary disease (COPD). Its pathogenesis is far from being completely understood. More than 40 yrs ago, investigators reported that the instillation of proteolytic enzymes in rats caused airspace enlargement [1] and that genetic deficiency in the neutrophil elastase inhibitor $\alpha_{1}$-antitripsin was also associated with emphysema [2]. These observations led to the hypothesis that emphysema was the result of an imbalance between proteolytic and antiproteolytic agents. The subsequent demonstration of increased numbers of neutrophils and macrophages in the lungs of smokers, and the known capacity of these cells to produce proteolytic enzymes, pointed to a connection between the inflammatory reaction characteristic of COPD and proteolytic/antiproteolytic imbalance, leading to the prevalent theory of an inflammatoryproteolytic origin of lung destruction in COPD. This theory does not, however, explain all the features in the lung of patients with COPD [3]. In recent years, alternative or complementary hypotheses have been proposed, including oxidative stress [4], apoptosis [5], cell senescence [6] and autoimmunity [7], providing a more complex and comprehensive view of the pathogenesis of emphysema in COPD.

Central to our current understanding of the pathogenesis of emphysema is the concept of a lung structure maintenance programme [8, 9], which considers that the lung is a metabolically active organ designed for gas exchange and the processing of endogenous substances constantly exposed to the environment. The lung's metabolic functions can only be guaranteed if the organ's structure is maintained and defended

*Dept of Pulmonary Medicine, Hospital Clínic-Institut d'Investigacions Biomèdiques August Pi i Sunyer, University of Barcelona, and ${ }^{\#}$ Centro de Investigación Biomédica en Red de Enfermedades Respiratorias, Barcelona, Spain.

CORRESPONDENCE: J.A. Barberà, Servei de Pneumologia, Hospital Clínic, Villarroel, 170, 08036 Barcelona, Spain. E-mail: jbarbera@clinic.ub.es against external and internal injuries. Defence and maintenance of the lung is achieved at various levels by means of antioxidants, antiproteases and the immune system, as well as stem cells that substitute apoptotic cells. In this scenario, destruction of the lung parenchyma may occur if there is ineffective antioxidation, unopposed proteolysis, excessive apoptosis, impaired clearance or substitution of apoptosed cells, cellular senescence without adequate cell replacement, or a switch from an innate toward an autoimmune response. Accordingly, the pathogenesis of emphysema can be broadly viewed as the disruption of the lung structure maintenance programme through various mechanisms that may synergise. This comprehensive concept may include and frame the various theories proposed for the pathobiology of emphysema.

In the process of lung destruction, both epithelial and endothelial cells die, and their apoptosis has been shown in human and animal lungs [10-12], suggesting that apoptosis may play a role in the pathogenesis of emphysema [5]. 10 yrs ago, investigators drew attention to the loss of alveolar capillary endothelial cells as a crucial event in the pathogenesis of emphysema [12]. Survival of endothelial cells is largely achieved through the action of vascular endothelial growth factor (VEGF), which is abundantly expressed in the lung [13]. Circumstantial and experimental evidence suggests that VEGF deficiency may play a role in the pathogenesis of emphysema in COPD patients. Emphysematous lungs of patients with advanced disease show decreased VEGF mRNA and protein expression, and decreased VEGF receptor 2 (VEGF-R2) [12]. Cigarette smoke extract reduces VEGF production in epithelial cells [14], and exposure to cigarette smoke causes significant decreases in VEGF and VEGF-R2 expression in rodent models of emphysema [15]. Furthermore, treatment of rats with the VEGF receptor blocker SU5416 induces alveolar septal cell apoptosis, pruning of the pulmonary arterial tree and enlargement of the air spaces [16], while the genetic deletion of VEGF [17] or the generation of anti-endothelial cell antibodies (including antibodies against VEGF-R2) [18] cause airspace enlargement. Accordingly, emphysema appears as a state of VEGF deficiency that compromises the survival of endothelial cells and, hence, the lung structure maintenance programme.

The mechanisms responsible for the reduced VEGF gene and protein expression in the lungs of COPD patients are poorly understood. In this issue of the European Respiratory Journal, YASUO et al. [19] investigate the role of the hypoxia inducible 
factor (HIF)-1 $\alpha$ in VEGF deficiency in COPD. HIF-1 $\alpha$ is a major factor regulating VEGF gene transcription [20]. The article by YASUO et al. [19] shows reduced protein expression of HIF-1 $\alpha$ in the lungs of patients with COPD, particularly in those with more severe disease. In their study, expression of HIF-1 $\alpha$ was related to the degree of airflow obstruction, as well as to the expression of VEGF and some downstream genes. These findings are important, since they provide a mechanism for the reduction of VEGF in emphysematous lungs that was not previously apparent. However, they are surprising because HIF- $1 \alpha$ is upregulated by hypoxia and inflammation [20], which are major features of advanced COPD. At first glance, one would have expected the opposite. To solve this apparent contradiction, YASUO et al. [19] explored the expression of histone deacetylase (HDAC) 2 and p53 in the lungs of their patients. They investigated these molecules because inhibition of HDAC downregulates hypoxia response genes and hypoxia-induced angiogenesis by suppressing HIF- $1 \alpha$ activity [21]. Such an inhibitory effect on HIF-1 $\alpha$ activity impedes the induction of VEGF in response to hypoxia, at both transcriptional and translational levels [21]. The expression of the proapoptotic tumour suppressor protein p53 is increased in lungs of COPD patients [22] and p53 may also contribute to regulating the transcription of the HIF- $1 \alpha$ gene.

Previous studies have shown that the levels and activities of HDAC are decreased in response to oxidative stress and exposure to cigarette smoke $[23,24]$. Since these factors are involved in the pathogenesis of COPD, decreased HDAC activity could eventually explain the reduction of VEGF expression in emphysema. Furthermore, HDAC2 negatively regulates p53 activity by inhibiting p53-DNA binding. Accordingly, the decrease in HDAC activity and, consequently, the upregulation of p53 could promote cell apoptosis. YASUO et al. [19] demonstrate reduced expression of HDAC2 and increased expression of p53 in patients with severe emphysema. As oxidative stress impairs the expression of HDAC2 [25] and upregulates p53 [26], the authors suggest that oxidative stress elicited directly by cigarette smoke or inflammation products might be at the origin of the reduction in HIF-1 $\alpha$, and that of VEGF, in emphysematous lungs [19].

The hypothesis proposed by YASUO et al. [19] is interesting as it links oxidative stress, a well-established mechanism of lung damage [4], with the impairment of VEGF, a critical factor for endothelial survival, and, therefore, the smooth running of the lung maintenance programme. However, single time-point observations do not provide mechanistic data and single correlations do not prove causality. The overall picture is far more complex. The authors propose oxidative stress as the mechanism for HDAC2 reduction, but oxidative stress is also related to the degree of inflammation, which in turn may induce HIF-1 $\alpha$ through the action of growth factors and cytokines [20, 27]. Unfortunately, arterial blood gases were not measured and, therefore, there are no data in the study by YASUO et al. [19] on the potential role of hypoxaemia, a major factor regulating HIF- $1 \alpha$ expression, in these complex interactions. Furthermore, it remains to be seen whether the reduced expression of HIF- $1 \alpha$ affects the lung exclusively or whether it is a more widespread phenomenon. The latter does not seem to be the case, since lung tumours express HIF-1 $\alpha$ [28] and patients with COPD may also show increased expression of
HIF- $1 \alpha$ in skeletal muscle [29]. It should also be noted that, if downregulation of HIF- $1 \alpha$ is the origin of decreased levels of VEGF and accounts for the development of emphysema, HIF- $1 \alpha$ should be reduced in the early stages of the disease. The latter was not the case in the group of patients with mild COPD in the study by YASUO et al. [19], who had HIF-1 $\alpha$ protein levels similar to that observed in the control group. There is also evidence that VEGF may be upregulated in pulmonary muscular arteries of patients with mild COPD [30]. Therefore, the hypothesis proposed by YASUO et al. [19] in their article requires the support of experimental studies addressed to test whether HIF- $1 \alpha$ suppression results in reduction of VEGF and subsequent development of pulmonary emphysema.

Despite the many questions pending solution, the article by YASUO et al. [19] represents an important advance in our understanding of the pathobiology of emphysema at the level of gene transcription of factors critically relevant to cell survival. The hypothesis proposed by the authors is linked to tissue damage induced by oxidative stress and it is framed within the concept of a disrupted lung maintenance programme. Better knowledge of the specific mechanisms involved in the pathogenesis of emphysema, and their place in a wider context where various pathways may converge and interact, will eventually provide the clues for therapeutic interventions capable of repairing lung destruction in COPD.

\section{STATEMENT OF INTEREST}

None declared.

\section{REFERENCES}

1 Gross P, Babyak MA, Tolker E, et al. Enzymatically produced pulmonary emphysema; a preliminary report. J Occup Med 1964; 6: 481-484.

2 Eriksson S. Pulmonary emphysema and $\alpha_{1}$-antitrypsin deficiency. Acta Med Scand 1964; 175: 197-205.

3 Chung KF, Adcock IM. Multifaceted mechanisms in COPD: inflammation, immunity, and tissue repair and destruction. Eur Respir J 2008; 31: 1334-1356.

4 MacNee W. Pathogenesis of chronic obstructive pulmonary disease. Proc Am Thorac Soc 2005; 2: 258-266.

5 Shapiro SD. Vascular atrophy and VEGFR-2 signaling: old theories of pulmonary emphysema meet new data. J Clin Invest 2000; 106: 1309-1310.

6 Aoshiba K, Nagai A. Senescence hypothesis for the pathogenetic mechanism of chronic obstructive pulmonary disease. Proc Am Thorac Soc 2009; 6: 596-601.

7 Cosio MG, Saetta M, Agusti A. Immunologic aspects of chronic obstructive pulmonary disease. N Engl J Med 2009; 360: 2445-2454.

8 Taraseviciene-Stewart L, Voelkel NF. Molecular pathogenesis of emphysema. J Clin Invest 2008; 118: 394-402.

9 Tuder RM, Yoshida T, Fijalkowka I, et al. Role of lung maintenance program in the heterogeneity of lung destruction in emphysema. Proc Am Thorac Soc 2006; 3: 673-679.

10 Calabrese F, Giacometti C, Beghe B, et al. Marked alveolar apoptosis/proliferation imbalance in end-stage emphysema. Respir Res 2005; 6: 14

11 Hodge S, Hodge G, Holmes M, et al. Increased airway epithelial and T-cell apoptosis in COPD remains despite smoking cessation. Eur Respir J 2005; 25: 447-454.

12 Kasahara Y, Tuder RM, Cool CD, et al. Endothelial cell death and decreased expression of vascular endothelial growth factor and 
vascular endothelial growth factor receptor 2 in emphysema. Am J Respir Crit Care Med 2001; 163: 737-744.

13 Voelkel NF, Vandivier RW, Tuder RM. Vascular endothelial growth factor in the lung. Am J Physiol Lung Cell Mol Physiol 2006; 290: L209-L221.

14 Thaikoottathil JV, Martin RJ, Zdunek J, et al. Cigarette smoke extract reduces VEGF in primary human airway epithelial cells. Eur Respir J 2009; 33: 835-843.

15 Marwick JA, Stevenson CS, Giddings J, et al. Cigarette smoke disrupts VEGF165-VEGFR-2 receptor signaling complex in rat lungs and patients with COPD: morphological impact of VEGFR-2 inhibition. Am J Physiol Lung Cell Mol Physiol 2006; 290: L897-L908.

16 Kasahara Y, Tuder RM, Taraseviciene-Stewart L, et al. Inhibition of VEGF receptors causes lung cell apoptosis and emphysema. J Clin Invest 2000; 106: 1311-1319.

17 Tang K, Rossiter HB, Wagner PD, et al. Lung-targeted VEGF inactivation leads to an emphysema phenotype in mice. J Appl Physiol 2004; 97: 1559-1566.

18 Taraseviciene-Stewart L, Scerbavicius R, Choe KH, et al. An animal model of autoimmune emphysema. Am J Respir Crit Care Med 2005; 171: 734-742.

19 Yasuo M, Mizuno S, Kraskauskas D, et al. Hypoxia inducible factor-1 $\alpha$ in human emphysema lung tissue. Eur Respir J 2011; 37: 775-783.

20 Semenza GL. Pulmonary vascular responses to chronic hypoxia mediated by hypoxia-inducible factor 1. Proc Am Thorac Soc 2005; 2: $68-70$.

21 Mie LY, Kim SH, Kim HS, et al. Inhibition of hypoxia-induced angiogenesis by FK228, a specific histone deacetylase inhibitor, via suppression of HIF-1 $\alpha$ activity. Biochem Biophys Res Commun 2003; 300: 241-246.

22 Siganaki M, Koutsopoulos AV, Neofytou E, et al. Deregulation of apoptosis mediators p53 and bcl2 in lung tissue of COPD patients. Respir Res 2010; 11: 46.

23 Adenuga D, Yao H, March TH, et al. Histone deacetylase 2 is phosphorylated, ubiquitinated, and degraded by cigarette smoke. Am J Respir Cell Mol Biol 2009; 40: 464-473.

24 Ito K, Ito M, Elliott WM, et al. Decreased histone deacetylase activity in chronic obstructive pulmonary disease. $N$ Engl J Med 2005; 352: 1967-1976.

25 Adcock IM, Cosio B, Tsaprouni L, et al. Redox regulation of histone deacetylases and glucocorticoid-mediated inhibition of the inflammatory response. Antioxid Redox Signal 2005; 7: 144-152.

26 Liu B, Chen Y, St Clair DK. ROS and p53: a versatile partnership. Free Radic Biol Med 2008; 44: 1529-1535.

27 Dehne N, Brune B. HIF-1 in the inflammatory microenvironment. Exp Cell Res 2009; 315: 1791-1797.

28 Ioannou M, Papamichali R, Kouvaras E, et al. Hypoxia inducible factor-1 alpha and vascular endothelial growth factor in biopsies of small cell lung carcinoma. Lung 2009; 187: 321-329.

29 Jatta K, Eliason G, Portela-Gomes GM, et al. Overexpression of von Hippel-Lindau protein in skeletal muscles of patients with chronic obstructive pulmonary disease. J Clin Pathol 2009; 62: 70-76.

30 Santos S, Peinado VI, Ramirez J, et al. Enhanced expression of vascular endothelial growth factor in pulmonary arteries of smokers and patients with moderate chronic obstructive pulmonary disease. Am J Respir Crit Care Med 2003; 167: 1250-1256. 